\title{
Development of a Machine Learning Model with a Function of Amygdala for Rapid Image Processing
}

\author{
Caiye Fan ${ }^{1, *}$, Sunwoo Ko², Linyang Yan $^{1}$ \\ ${ }^{1}$ Department of Cultural Technology (Artificial Intelligence), Jeonju University, Korea \\ ${ }^{2}$ Department of Artificial Intelligence, Jeonju University, Korea \\ *Corresponding author: caiyefan@jj.ac.kr
}

\begin{abstract}
In this paper, a method of implementing convolutional neural network that can quickly deal with dangerous situations is designed based on the processing rules of the amygdala of human brain. By studying the processing rules of the amygdala in the human brain, we can understand neuron activity when humans are at risk, build similar models, and test relevant data. A neural network model can be constructed by changing structure, loss function and number of filters the general convolutional neural network model. The designed neural network model can quickly and accurately predict dangers. It was used to test data set and good results were obtained.
\end{abstract}

Keywords: Amygdala, biological learning method, convolutional neural network, image processing

\section{Introduction}

With the continuous development of technology, people have begun to use machines to do a lot of work. Using machines to identify dangers is one of the most important technologies that many developers are working on. People gather information from the outside world with their eyes and ears, and can easily identify an event, or determine whether it is dangerous, but this information to the machine is a bunch of zeros and ones.

For people, danger can be understood as a situation that can cause great losses and even cause people to lose their lives. It is of great importance to spot dangers accurately, but it is even more important to spot them quickly and make quick decisions.

As we all know, human brain is a very complex organ with many functions. One of the most important features of many functions is to handle dangerous situations. For example, when a person is walking down the road and suddenly a snake-like monster emerges, he or she tends not to be the first to judge what the monster in front of them is, but to be the first to fear and hide behind them. He or she would go the front of the monster to determine what it is after going back to a safe place. If he or she chooses to first observe the monster instead of escaping, it will be very likely to get bitten by the monster and thus fall into danger.

Developers have developed neural networks and convolutional neural networks based on models of human brain. But many developers only care about the accuracy of model predictions rather than going deeper into the human brain and testing whether the developed model is similar to the human brain.

It is necessary to delve into whether the neural network model has the same structure and processing as the human brain, and whether more brain functions can be added to the neural network model. Such researches can be used to refine existing models or develop a new model based on the human brain.

My goal is to build models of the brain functions through relevant researches. In this paper, what I have to do is to implement the brain functions for processing dangers into convolutional neural networks.

By changing the model construction, loss function, and the number of filters, a neural network model that can quickly and accurately determine the danger just as brain does is constructed. Through such a neural network, dangerous situations can be rapidly determined to reduce the losses caused by the danger. 


\section{Research of the Brain and $\mathrm{CNN}$}

\subsection{Research of the Brain}

The human brain receives information from the outside world through sensory organs such as eyes and ears, and then transmits and processes information in the brain through organs such as thalamus, cortex, amygdala and hippocampus. The connections between these organs form multiple circuits, and each function in the human brain consists of multiple circuits [1].

The information received by the brain through the sensory organs is collected in the thalamus, which acts as an intermediate station for the simple processing of information, and then such information is transmitted to cortex and other organs [2]. The cortex processes the information in detail and then transmits it to other organs, such as hippocampus and amygdala. The hippocampus is an organ related to memory storage [3], and amygdala has functions such as sensing danger and responding to dangerous situations [4].

Neurons and synapses play a very important role when the brain performs a certain function. All the information in the brain is transmitted through neurons and synapses [5]. Neurons are composed of nucleus, dendrites, and axons. The nucleus stores genetic material and provides the materials required for the survival of neurons. The dendrites act as input sites to receive information from the last neuron. The axons act as output sites to communicate information to the next neuron by generating chemicals. When a neuron receives a signal and is activated, it generates electrical propagation within the neuron, passing information from the dendrites to the axons for output. There is synaptic space between the two neurons. When information transmission occurs, the chemical substances generated by the dendrites are combined through synapses for information transmission [5], [6].

The amygdala, the most important organ of the brain for dealing with dangerous situations, can sense potential dangers, make judgments, and respond to perceived dangers. For example, a mouse will instantly become stiff and enter a state of suspended animation if it meets a cat, its natural enemy. This is the amygdala in the brain of the mouse that senses danger (cat) and reacts to danger (suspended animation).

The amygdala's perception of danger is divided into two categories: one is the perception of the innate danger from heredity, and the other is the perception of the danger of the brain by learning. We call it Fear Conditioning [7]. For example, a cat will identify an electric shock as dangerous and have a stiff body. Then, at the same time, the bell rings. After repeated learning, as long as the bell rings, the amygdala in the mouse brain will identify it as dangerous and the body will be stiff.

The amygdala constantly receives information but it ignores most of the information and only responds to potentially dangerous information. The amygdala simultaneously receives information from two sources, namely thalamus and cortex. The information obtained from sensory organs is collected in the thalamus. The thalamus simply processes the information and sends it to cortex for more detailed processing [8]. But at the same time, thalamus sends this information to the amygdala, which is known as low path.

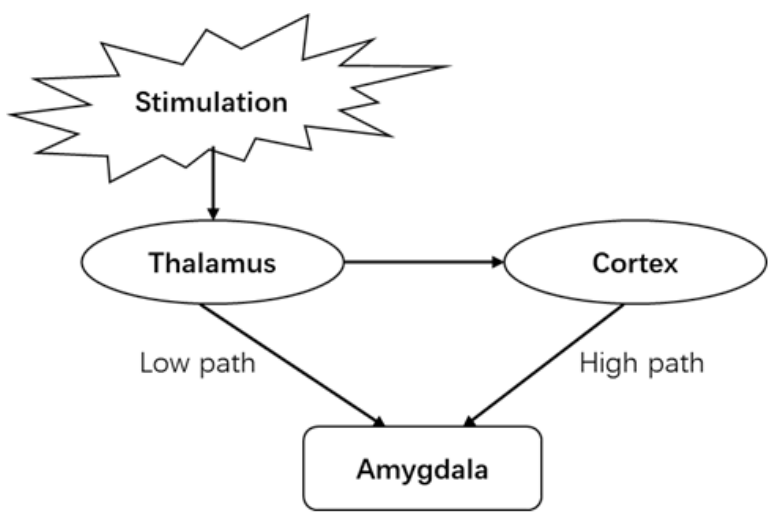

Figure 1 Transmission Path to the Amygdala.

The information transmitted by thalamus to cortex is processed through cortex and then transmitted to amygdala, which is known as high path. The information of the low path has not been processed in detail, so it can be quickly sent to amygdala. The amygdala can make dangerous judgments quickly, 
but relatively low accuracy. The information of the high path is processed by cortex in detail, and the speed of transmission to amygdala is relatively slow, but judgment is relatively accurate [9], [10], [11].

\subsection{Convolutional Neural Network}

Image recognition has always been an area of concern. Convolutional neural networks, as a neural network for image recognition, are more in line with the way human brains process visual information than ordinary image classification neural networks. When visual information such as a picture is transmitted into the human brain through eyes, the brain does not analyze the picture as a whole, but divides it into many small pieces, and local analysis on features such as contours, edges, textures is carried out in each small piece.

The convolutional neural network splits the picture into smaller pieces and is interested in the features within those small pieces. These features are collected by convolution operations on the filters in the model trained with the features as input.

The convolutional neural network is mainly composed of the Input layer, Convolutional Layer, Pooling Layer, and Fully-Connected Layer.

In the convolutional layer, the filter performs convolution on the input image by sliding the window. Each filter corresponds to a different feature. After the convolution of images by multiple filters, multiple feature images can be extracted. The resulting feature map is then sent to the pooling layer.

Max Pooling is a common pooling method used in the Pooling Layer. Max Pooling is to slide the window with a filter of a certain size, leaving only the largest value. Thus, the pooling layer compresses the feature map, extracts the main features, and makes the model less complicated to improve the calculation speed.

Finally, the compressed feature map is sent to the Fully-Connected layer to calculate the prediction result. When the model is trained, the loss function is calculated by the predicted result to train the weight of the Fully-Connected layer and each filter of the Convolution layer.

\section{Model Development and Experiment}

People have multiple sensory organs, and amygdala combines the information collected by various sensory organs to sense and identify dangers. But in this paper, I only model, experiment, and analyze visual senses.

\subsection{Model Development}

\subsubsection{Connection of Thalamus and Cortex}

As mentioned earlier, thalamus receives information from sensory organs and sends it to cortex for more detailed processing. The detailed information is transmitted to other organs, compared with the memory extracted from hippocampus, and compared to determine what is perceived by our sensory organs. The general Convolutional Neural Network is consistent with this, and the picture is extracted by the Convolution Layer, and then classified by Fully-Connected Layer.

So here, we use the general Convolutional Neural Network structure for modelling to obtain the filter needed to classify specified data set. These filters are like the cerebral cortex, which processes the information in detail and transmits the processed information to the next organ to further classify and identify the original data.

\subsubsection{Connection of Cortex and Amygdala}

In the brain, cerebral cortex processes information both for categorizing primitive objects and for perceiving danger. After cortex processes information in detail, it transmits the processed information to amygdala. Based on the processed information, it is perceived whether a person is in a dangerous environment.

In this part of the model, we again use the general convolutional neural network model, but we do not need to train the filters in the convolution layer again because in the previous link, we have obtained the filters to process specific data sets. By reusing the trained filters, the time for model training can be greatly reduced. 
In this part of the model, what we need to do is to determine whether it is dangerous or not. Therefore, the predicted results fall into two categories, namely dangerous and safe. When an error is predicted and a loss occurs, the model trains the weight by calculating the loss function. After the training is completed, we can get the weights in the Fully-Connected layer, which can be further used to input the information, and after calculation, a result indicating whether it is dangerous will be output.

\subsubsection{Connection of Thalamus and Amygdala}

The role of the connection between thalamus and amygdala is to quickly perceive danger through simple, unprocessed information.

In this part of the model, we do not retrain the model in the network model, but directly use the filters and weights that have been trained in the first two links. However, this link does not require complete detailed information processing, so we only select the filters with the highest risk performance, process the images, and then directly make dangerous judgments.

The complete model structure can be seen through the picture.

Thalamus-Cortex Connection Model

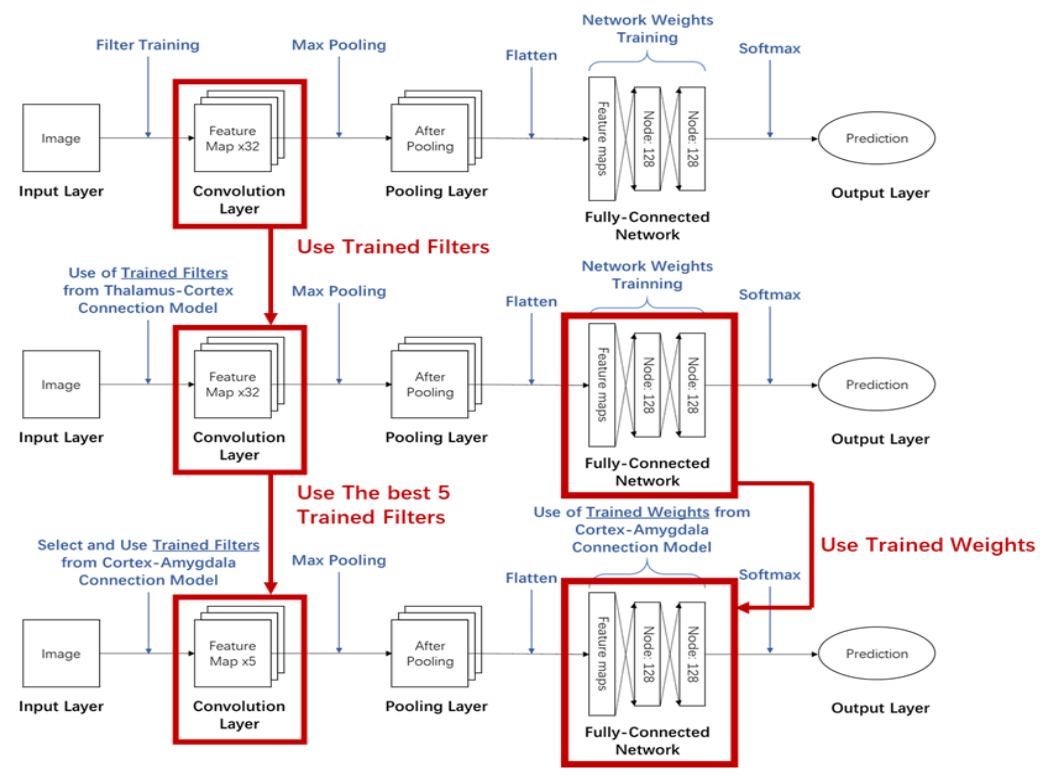

Figure 2 Model with a Function of Amygdala.

\subsection{Experimental Analysis}

The experiment used the CIFAR-10 dataset and selected five kinds of animals to treat 'dog' as a dangerous animal. The size of the data is $28 \times 28$. The size of the data set is 20,000 training data, 5,000 test data, and 5,000 verification data.

When we train the model to determine whether it is a danger, the general loss function is:

$$
\text { Loss }=\sum_{t}\left(y_{i}-t_{i}\right)^{2}
$$

And when analyzing prediction errors, we can see from the picture that the prediction errors can be divided into two types. The Type1 Error determines that safety is dangerous, and Type 2 Error indicates that dangerous situation is safe. In reality, if Type 2 Error occurs, that is, when we judge the dangerous situation as safe, there may be a major loss or even loss of life. But looking at the loss function in the model, we find that when both Type1 Error and Type 2 Error occur, the loss of the same amount will occur.

So here I will make a change to the loss function:

$$
\text { Loss }=\alpha \operatorname{Loss}_{\text {type } 2}+(1-\alpha) \text { Loss }_{\text {type } 1}
$$




$$
\text { Loss }=\alpha \sum_{i \in \text { type } 2}\left(y_{i}-t_{i}\right)^{2}+(1-\alpha) \sum_{i \in \text { type } 1}\left(y_{i}-t_{i}\right)^{2}
$$

By adjusting the size, the size of Type2 Error loss is changed. The purpose is to produce a greater loss when Type 2 Error occurs. Test set multiple levels of $\mathrm{x}$ for testing, and the larger the value, the greater the loss caused by Type2 Error.

Table 1 Model Experiment Result.

\begin{tabular}{|c|c|c|c|}
\hline \multirow{2}{*}{ Alpha } & Model & $\begin{array}{c}\text { Danger data } \\
\text { (size: 10) }\end{array}$ & $\begin{array}{c}\text { Not danger data } \\
\text { (size: } 90)\end{array}$ \\
\hline \multirow{2}{*}{0.7} & Full & $40 \%$ & $94 \%$ \\
\cline { 2 - 4 } & Quick & $60 \%$ & $46 \%$ \\
\cline { 2 - 4 } 0.75 & Full & $60 \%$ & $94 \%$ \\
\hline \multirow{2}{*}{0.8} & Quick & $90 \%$ & $36 \%$ \\
\cline { 2 - 4 } & Full & $50 \%$ & $89 \%$ \\
\hline \multirow{2}{*}{0.85} & Quick & $80 \%$ & $58 \%$ \\
\cline { 2 - 4 } & Full & $70 \%$ & $87 \%$ \\
\hline \multirow{2}{*}{0.9} & Quick & $100 \%$ & $24 \%$ \\
\cline { 2 - 4 } & Full & $60 \%$ & $95 \%$ \\
\hline \multirow{2}{*}{0.95} & Quick & $100 \%$ & $8 \%$ \\
\cline { 2 - 4 } & Full & $80 \%$ & $95 \%$ \\
\hline
\end{tabular}

The training results are shown in Figure 2. As can be seen from the figure, when the alpha increases, the model becomes more sensitive to dangerous data, and the judgment result of dangerous data is continuously increased.

In Figure 2, 'full' means a model that uses all the filters, and 'quick' means a simple model that uses only five filters. Comparing the test results of the two models, the quick model has more than fullhigh-risk sensitivity, which is in line with people's quick judgment and response to dangerous situations.

Table 2 Time Required for Each Model.

\begin{tabular}{|c|c|c|c|}
\hline \multicolumn{2}{|c|}{} & \multicolumn{2}{|c|}{ Cost Time } \\
\cline { 3 - 3 } \multicolumn{2}{|c|}{ Model } & Training (1 epoch) & Test (1 data) \\
\hline \multirow{2}{*}{ full model } & Connection 1 & $23 \mathrm{sec} / 1$ epoch & \multirow{2}{*}{$0.0579 \mathrm{sec} / 1$ data } \\
\cline { 2 - 3 } & Connection 2 & $17 \mathrm{sec} / 1$ epoch & $0.0568 \mathrm{sec} / 1$ data \\
\hline quick model & Connection 3 & x & 0 \\
\hline
\end{tabular}

Table 2 shows the time required for each model training and testing, where Connection1, 2, and 3 are the three connections between thalamus, cortex, and amygdala, respectively. We can see that the time required for the full model and the quick model test is almost the same, but the quick model does not need to be trained, so it can save a lot of time. And the advantage of the quick model is that it is more sensitive to danger than the full model, preventing the loss as much as possible.

\section{Conclusion}

In this paper, we established the connection model of sensory organs, thalamus, cortex, amygdala, and hippocampus by studying human brain. The neural network model with the function of amygdala risk processing was realized by the convolutional neural network. The experiment was carried out by the CIFA-10 data set, and the expected results are obtained.

The main purpose of this paper is not to get a high-precision model of the test results, but to study how to make the model as sensitive to danger as the human brain. It can better prevent the huge loss caused by Type2 Error.

The human brain has multiple sensory organs, and gathers information from various organs to more accurately distinguish things and perceive dangers. In this paper, so far only vision has been studied 
and experimented with. In future studies, I hope to combine human sensory organs, such as ear, to refine the model of danger perception, making the model more dangerously treated like the amygdala of the brain.

\section{References}

[1] J. L. Hellier, The Brain, the Nervous System, and Their Diseases [3 volumes]; ABC-CLIO, 2014, pp. 300-303.

[2] M. S. Gazzaniga, G. R. Mangun, R. B. Ivry, Cognitive Neuroscience: The Biology of the Mind; New York: W.W. Norton, 2014, pp. 45

[3] L. R. Squire, "Memory and the hippocampus: a synthesis from findings with rats, monkeys, and humans," Psychol Rev, vol. 99, pp. 195-231, April 1992.

[4] J. S. Feinstein, R. Adolphs, A. Damasio, D. Tranel, "The human amygdala and the induction and experience of fear," Current Biology, vol. 21, pp. 34-38, January 2011.

[5] E. R. Kandel, J. H. Schwartz, T. M. Jessel, H. James, M. Thomas, Principles of Neural Science; New York: McGraw-Hill, 2000, ch. 2-4.

[6] J. E. LeDoux, Synaptic Self: How Our Brains Become Who We Are also viewed; Penguin, 2003, ch. 3, pp.80-117.

[7] J. J. Kim, M. W. Jung, "Neural circuits and mechanisms involved in Pavlovian fear conditioning: a critical review," Neurosci Biobehav Rev, vol. 30, pp. 188-202, February 2006.

[8] X. F. Li, G. E. Stutzmann, J. E. LeDoux, "Convergent but temporally separated inputs to lateral amygdala neurons from the auditory thalamus and auditory cortex use different postsynaptic receptors: in vivo intracellular and extracellular recordings in fear conditioning pathways," Learn Mem, vol. 3, pp. 229-242, September 1996.

[9] G. J. Quirk, J. L. Armony, J. E. LeDoux, "Fear Conditioning Enhances Different Temporal Components of Tone-Evoked Spike Trains in Auditory Cortex and Lateral Amygdala, "Neuron, vol. 19, pp. 613-624, September 1997.

[10] G. J. Quirk, C. Repa, J. E. LeDoux, "Fear conditioning enhances short-latency auditory responses of lateral amygdala neurons: parallel recordings in the freely behaving rat," Neuron, vol. 15, pp. 102939, November 1995.

[11] J. E. LeDoux, Synaptic Self: How Our Brains Become Who We Are also viewed; Penguin, 2003, ch. 5 , pp.210-218. 